\title{
A New High-Rate Differential Space-Time Block Coding Scheme
}

\author{
Naofal Al-Dhahir, Senior Member, IEEE
}

\begin{abstract}
A new high-rate differential space-time transmission scheme based on spatial multiplexing of Alamouti-encoded information streams is developed in this letter. At the receiver, joint space-time differential interference cancellation and decoding is performed, realizing diversity and rate gains, without requiring channel knowledge or bandwidth expansion. Our focus is on the case of two information streams with two transmit antennas per stream on flat-fading channels for simplicity. However, the development readily extends to more than two information streams, to more than two transmit antennas per stream, and to frequency-selective channels using previously published techniques.
\end{abstract}

Index Terms-Differential encoding, interference cancellation, space-time block code (STBC), spatial multiplexing.

\section{INTRODUCTION}

$\mathbf{T}$ HE ALAMOUTI space-time block code (STBC) for two transmit antennas enjoys the following three main attractive features: 1) it does not require channel knowledge at the transmitter (i.e. operates open loop); 2) it achieves full (i.e., second-order) spatial diversity gains with a single receive antenna (which makes it attractive for the downlink); and 3) its maximum-likelihood decoder performs only linear processing (due to the orthogonal spatio-temporal structure of the code). On the other hand, the Alamouti STBC has two main limitations. First, it requires channel knowledge at the receiver which can be acquired using training sequences at the expense of rate overhead. For high-mobility broadband scenarios (such as the forthcoming fourth-generation multimedia wireless systems), it becomes very costly and difficult in practice to estimate and track the large number of channel parameters ${ }^{1}$ accurately resulting in significant performance degradation. The second limitation of the Alamouti STBC is its achievable rate. It transmits two information symbols over two symbol periods using two transmit antennas resulting in a rate of one, which is half the maximum possible rate of two achieved for this scenario using spatial multiplexing. This rate loss is due to the two inserted redundant symbols that allow this code to achieve full spatial diversity gains. There is a fundamental tradeoff between rate and diversity in a multiple-antenna wireless system. With one re-

Manuscript received April 6, 2003. The associate editor coordinating the review of this paper and approving it for publication was Dr. J. Ritcey.

The author is with the University of Texas at Dallas, Richardson TX 75083 USA (e-mail: aldhahir@utdallas.edu).

Digital Object Identifier 10.1109/LCOMM.2003.820095

${ }^{1}$ The number of channel parameters increases linearly with the number of transmit/receive antennas and with the ratio of channel delay spread to symbol period. ceive antenna, the Alamouti STBC achieves the optimal rate-diversity tradeoff possible. Hence, it is not possible to increase its rate without sacrificing diversity.

A differential form of the Alamouti Rate-1 STBC was proposed in [1] which obviates the need for channel estimation at the receiver without sacrificing diversity gains. The price paid is an signal-to-noise ratio (SNR) loss of $3 \mathrm{~dB}$ relative to coherent decoding (with perfect channel estimation). This penalty might be acceptable under high-mobility conditions where channel estimation errors could result in more than 3-dB SNR loss in addition to the complexity and power consumption of the channel estimation module in the user terminal. A doubling of the achievable rate in the Alamouti scheme, without sacrificing precious RF bandwidth, can be achieved through a parallel, co-channel, and synchronous transmission of two Alamouti space-time-encoded signals using four transmit antennas. Only one extra receive antenna is needed to separate the two streams using the linear spatio-temporal interference cancellation scheme in [2].

Our objective in this paper is to combine the benefits of the two schemes in [1] and [2] by developing a Rate-2 differential Alamouti STBC scheme and the associated differential space-time joint interference cancellation and decoding algorithms.

\section{BACKGROUND}

\section{A. Channel Model and Assumptions}

We consider a transmission scenario where there are four transmit and two receive antennas. The four transmitted signals are generated using two Alamouti STBC encoders [3] operating in parallel on two cochannel (i.e. sharing the same frequency band) and synchronized (i.e. transmitted simultaneously over the same time duration) signals $\left\{x_{1}(k)\right\}$ and $\left\{s_{1}(k)\right\}$ which are, in turn, generated from differential encoding of two independent information streams $\left\{u^{x}(k)\right\}$ and $\left\{u^{s}(k)\right\}$ each at rate $\mathrm{R}$.

The channel from transmit antenna $i$ (for $i=1,2$ ) to receive antenna $j$ (for $j=1,2$ ) is assumed flat-fading and its coefficient is denoted by $h_{i, j}$ for the first stream and by $g_{i, j}$ for the second stream (with a single receive antenna, we drop the second index in the subscript). Both $h_{i, j}$ and $g_{i, j}$ are assumed complex circularly-symmetric i.i.d. Gaussian random variables with zero mean and unit variance. The noise at receive antenna $j$ (for $j=1,2$ ) at time $k$ is denoted by $z_{j}(k)$ which is modeled as a complex circularly-symmetric zero-mean Gaussian random variable. 


\section{B. Rate-1 Alamouti STBC}

With two transmit antennas, the Alamouti STBC [3] groups the input symbols into pairs, each denoted by a vector $\mathbf{x}(k) \stackrel{\text { def }}{=}$ $\left[\begin{array}{lll}x_{1}(k) & x_{2}(k)\end{array}\right]^{T}$ (where $(\cdot)^{T}$ denotes the transpose), which are processed by the space-time block encoder according to the rule

$$
\begin{aligned}
\mathbf{x}(k) & =\left[\begin{array}{l}
x_{1}(k) \\
x_{2}(k)
\end{array}\right] \longrightarrow\left[\begin{array}{cc}
x_{1}(k) & x_{1}(k+1) \\
x_{2}(k) & x_{2}(k+1)
\end{array}\right] \\
& =\left[\begin{array}{ccc}
x_{1}(k) & x_{1}(k+1) \\
-\bar{x}_{1}(k+1) & \bar{x}_{1}(k)
\end{array}\right] \vec{\downarrow} \text { time }
\end{aligned}
$$

where (.) denotes the complex-conjugate transpose operation. Assuming a single receive antenna, the received signals over two consecutive symbol time instants, denoted by $y(k)$ and $y(k+1)$, are

$$
\mathbf{y}(k) \stackrel{\text { def }}{=}\left[\begin{array}{c}
y(k) \\
-\bar{y}(k+1)
\end{array}\right]=\mathbf{H}_{Q} \mathbf{x}(k)+\mathbf{z}(k)
$$

where

$$
\mathbf{H}_{Q} \stackrel{\text { def }}{=}\left[\begin{array}{cc}
h_{1} & h_{2} \\
-\bar{h}_{2} & \bar{h}_{1}
\end{array}\right] ; \mathbf{z}(k)=\left[\begin{array}{c}
z(k) \\
-\bar{z}(k+1)
\end{array}\right]
$$

where we assumed that the two channels are constant over the transmission of two symbols. The orthogonal channel matrix $\mathbf{H}_{Q}$ in (1) belongs to a class of $2 \times 2$ complex matrices known as quaternions and will be denoted by the subscript $(.)_{Q}$ in this paper. In addition to their orthogonality, the set of quaternions is closed under addition, multiplication, and inversion.

\section{NeW DifFEREnTIAl RATE-2 STBC}

Write (1) in the following quaternionic form ${ }^{2}$

$$
\begin{aligned}
& {\left[\begin{array}{cc}
y(k) & y(k+1) \\
-\bar{y}(k+1) & \bar{y}(k)
\end{array}\right]} \\
& =\left[\begin{array}{cc}
h_{1} & h_{2} \\
-\bar{h}_{2} & \bar{h}_{1}
\end{array}\right]\left[\begin{array}{cc}
x_{1}(k) & x_{1}(k+1) \\
-\bar{x}_{1}(k+1) & \bar{x}_{1}(k)
\end{array}\right] \\
& \quad+\left[\begin{array}{cc}
z(k) & z(k+1) \\
-\bar{z}(k+1) & \bar{z}(k)
\end{array}\right] .
\end{aligned}
$$

Ignoring noise, we can write (2) compactly as

$$
\mathbf{Y}_{Q}(k: k+1)=\mathbf{H}_{Q} \mathbf{X}_{Q}(k: k+1)
$$

where an argument $\left(l_{1}: l_{2}\right)$ for a matrix indicates that it contains symbols with time indices ranging from $l_{1}$ to $l_{2}$. With two co-channel synchronized Alamouti STBC encoders with independent information streams $\left\{x_{1}(k)\right\}$ and $\left\{s_{1}(k)\right\}$ and 2 receive antennas, (3) becomes

$$
\left[\begin{array}{l}
\mathbf{Y}_{Q}^{(1)}(k: k+1) \\
\mathbf{Y}_{Q}^{(2)}(k: k+1)
\end{array}\right]=\left[\begin{array}{ll}
\mathbf{H}_{Q}^{(1)} & \mathbf{G}_{Q}^{(1)} \\
\mathbf{H}_{Q}^{(2)} & \mathbf{G}_{Q}^{(2)}
\end{array}\right]\left[\begin{array}{c}
\mathbf{X}_{Q}(k: k+1) \\
\mathbf{S}_{Q}(k: k+1)
\end{array}\right]
$$

Assume that the channel matrices $\mathbf{H}_{Q}^{(1)}, \mathbf{H}_{Q}^{(2)}, \mathbf{G}_{Q}^{(1)}, \mathbf{G}_{Q}^{(2)}$ are constant over four consecutive symbols with time indices $k$ to $k+3$. Then

$$
\begin{aligned}
{\left[\begin{array}{c}
\mathbf{Y}_{Q}^{(1)}(k+2: k+3) \\
\mathbf{Y}_{Q}^{(2)}(k+2: k+3)
\end{array}\right] } & \\
& =\left[\begin{array}{ll}
\mathbf{H}_{Q}^{(1)} & \mathbf{G}_{Q}^{(1)} \\
\mathbf{H}_{Q}^{(2)} & \mathbf{G}_{Q}^{(2)}
\end{array}\right]\left[\begin{array}{c}
\mathbf{X}_{Q}(k+2: k+3) \\
\mathbf{S}_{Q}(k+2: k+3)
\end{array}\right] .
\end{aligned}
$$

${ }^{2}$ This is an alternative derivation (taken from [4]) of the differential Alamouti scheme first proposed in [1].

$$
\begin{aligned}
& \text { Combining (4) and (5), we get } \\
& \underbrace{\left[\begin{array}{cc}
\mathbf{Y}_{Q}^{(1)}(k+2: k+3) & \mathbf{Y}_{Q}^{(1)}(k: k+1) \\
\mathbf{Y}_{Q}^{(2)}(k+2: k+3) & \mathbf{Y}_{Q}^{(2)}(k: k+1)
\end{array}\right]}_{\tilde{\mathbf{Y}}(k: k+3)} \\
& =\left[\begin{array}{ll}
\mathbf{H}_{Q}^{(1)} & \mathbf{G}_{Q}^{(1)} \\
\mathbf{H}_{Q}^{(2)} & \mathbf{G}_{Q}^{(2)}
\end{array}\right]\left[\begin{array}{ll}
\mathbf{X}_{Q}(k+2: k+3) & \mathbf{X}_{Q}(k: k+1) \\
\mathbf{S}_{Q}(k+2: k+3) & \mathbf{S}_{Q}(k: k+1)
\end{array}\right] .
\end{aligned}
$$

It is critical to note here that, unlike the Rate-1 case in (3) where the equivalent channel matrix is a quaternion, it is not a quaternion in the Rate- 2 case in (6). Instead, it is a $4 \times 4$ matrix consisting of four quaternions each corresponding to an information stream/receive-antenna pair. Therefore, a standard differential detection rule will not work. However, we show next that by doubling the observation interval length, over which the channels are assumed fixed, from four (as in the differential Rate-1 STBC case) to eight symbols, it is possible to decode both information streams simultaneously and differentially with spatial diversity gains for both. Consider the following differential space-time encoding rule ${ }^{3}$

$$
\begin{aligned}
& \underbrace{\left[\begin{array}{cc}
\mathbf{X}_{Q}(k+2: k+3) & \mathbf{X}_{Q}(k: k+1) \\
\mathbf{S}_{Q}(k+2: k+3) & \mathbf{S}_{Q}(k: k+1)
\end{array}\right]}_{\tilde{\mathbf{X}}(k: k+3)} \\
& =\underbrace{\left[\begin{array}{cc}
\mathbf{X}_{Q}(k-2: k-1) & \mathbf{X}_{Q}(k-4: k-3) \\
\mathbf{S}_{Q}(k-2: k-1) & \mathbf{S}_{Q}(k-4: k-3)
\end{array}\right]}_{\tilde{\mathbf{X}}(k-4: k-1)} \\
& \times \underbrace{\left[\begin{array}{cc}
\mathbf{U}_{Q}^{x}(k+2: k+3) & \mathbf{U}_{Q}^{x}(k: k+1) \\
\mathbf{U}_{Q}^{s}(k+2: k+3) & \mathbf{U}_{Q}^{s}(k: k+1
\end{array}\right]}_{\tilde{\mathbf{U}}(k: k+3)}
\end{aligned}
$$

where $\tilde{\mathbf{U}}(k: k+3)$ contains the eight information symbols (four from each information stream corresponding to symbol instants $k$ to $k+3$ ) to be detected. Now, assume that the four channel matrices $\mathbf{H}_{Q}^{(1)}, \mathbf{G}_{Q}^{(1)}, \mathbf{H}_{Q}^{(2)}$, and $\mathbf{G}_{Q}^{(2)}$ are constant over the eight consecutive symbol instants $k-4$ to $k+3 .{ }^{4}$ Then, (6) can be expressed recursively as follows

$$
\begin{aligned}
\tilde{\mathbf{Y}}(k & : k+3) \\
= & {\left[\begin{array}{ll}
\mathbf{H}_{Q}^{(1)} & \mathbf{G}_{Q}^{(1)} \\
\mathbf{H}_{Q}^{(2)} & \mathbf{G}_{Q}^{(2)}
\end{array}\right] \tilde{\mathbf{X}}(k-4: k-1) \tilde{\mathbf{U}}(k: k+3) } \\
= & \underbrace{\left[\begin{array}{cc}
\mathbf{Y}_{Q}^{(1)}(k-2: k-1) & \mathbf{Y}_{Q}^{(1)}(k-4: k-3) \\
\mathbf{Y}_{Q}^{(2)}(k-2: k-1) & \mathbf{Y}_{Q}^{(2)}(k-4: k-3)
\end{array}\right]}_{\tilde{\mathbf{Y}}(k-4: k-1)} \\
& \times \tilde{\mathbf{U}}(k: k+3) .
\end{aligned}
$$

This leads to the following differential space-time ML ${ }^{5}$ detection metric which is minimized over all signal constellation symbols $^{6}$

$$
\|\tilde{\mathbf{Y}}(k: k+3)-\tilde{\mathbf{Y}}(k-4: k-1) \tilde{\mathbf{U}}(k: k+3)\|^{2} .
$$

${ }^{3}$ This rule implies that the two streams must be coordinated.

${ }^{4}$ For a Rate- $L$ differential STBC, the channels need to be assumed constant over $4 L$ consecutive symbol instants.

${ }^{5}$ Assuming the noise white Gaussian.

${ }^{6} \mathrm{It}$ is also possible to do ML detection with 1 receive antenna. 


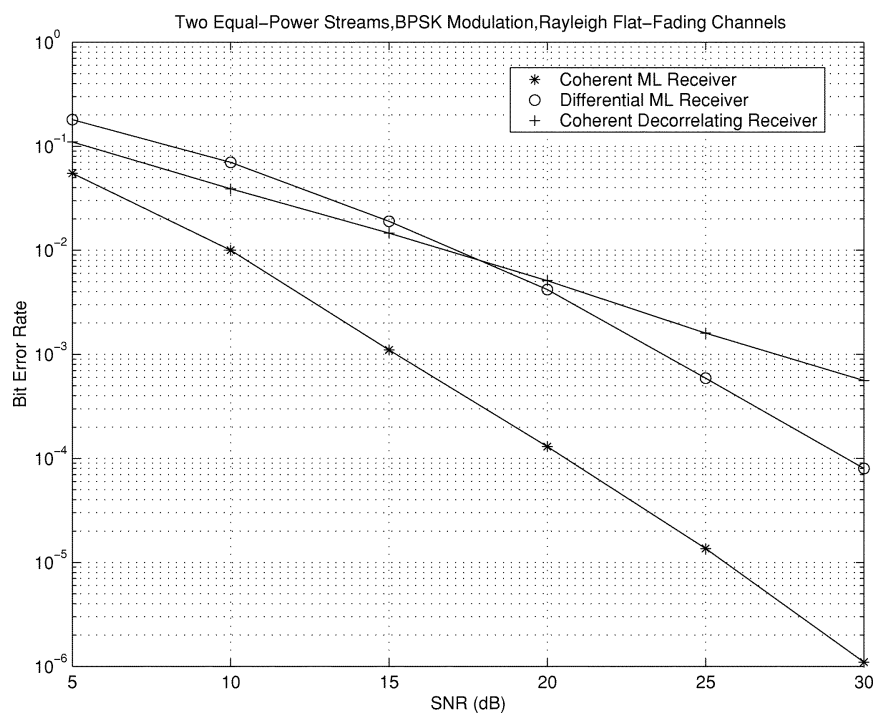

Fig. 1. BER of proposed scheme.

Because of the block quaternionic structure of the involved matrices, we only need to minimize the norms of two (instead of all four) columns in (9). This leads to two exhaustive searches each of size $M^{4}$. In general, the complexity of this ML detector grows exponentially with the signal constellation size, information rate, and number of transmit antennas per information stream. We can apply other space-time reduced-complexity detection algorithms such as the zero-forcing, MMSE decorrelating, decision feedback, or sphere decoders to (8).

Extensions of the presented scheme to the Rate- $L$ case and to more than two transmit antennas per stream are straightforward and follow the methodology in [5]. In addition, our scheme can be readily extended to frequency-selective channels with Rate- $L$ using the approach in [4] under the assumption that the channels are fixed over $4 L$ consecutive transmission blocks (not symbols). Clearly, this assumption becomes less accurate as the Doppler rate, block length, and $L$ increase leading to an error floor.

\section{Simulation Results}

Fig. 1 compares the performance of our differential Rate-2 STBC scheme with coherent ML and decorrelating schemes, assuming perfect channel knowledge at the receiver. The figure shows that the performance gap between the coherent and differential ML receivers is more than the 3-dB gap observed in the Rate-1 case (due to presence of inter-stream interference). This gap is reduced when taking into account the effects of practical channel estimation on performance of the coherent scheme. It is also interesting to note that the proposed differential ML detector outperforms the coherent decorrelating detector at high SNR (even with perfect channel knowledge for the latter!). This occurs because the coherent decorrelating receiver achieves only second (not fourth) diversity order for each stream since the second receive antenna is used to null the other stream.

\section{ACKNOWLEDGMENT}

The author thanks Dr. Suhas Diggavi of EPFL Switzerland, for several stimulating discussions.

\section{REFERENCES}

[1] V. Tarokh and H. Jafarkhani, "A differential detection scheme for transmit diversity," IEEE J. Select. Areas Commun., vol. 18, pp. 1169-1174, July 2000.

[2] A. F. Naguib, N. Seshadri, and A. R. Calderbank, "Applications of space-time block codes and interference suppression for high capacity and high data rate wireless systems," in Proc. 32nd Asilomar Conf. on Signals, Systems and Computers, 1998, pp. 1803-1810.

[3] S. Alamouti, "A simple transmit diversity technique for wireless communications," IEEE J. Select. Areas Commun., vol. 16, pp. 1451-1458, Oct. 1998.

[4] S. Diggavi, N. Al-Dhahir, A. Stamoulis, and A. R. Calderbank, "Differential space-time coding for frequency-selective channels," IEEE Commun. Lett., vol. 6, pp. 253-255, June 2002.

[5] A. Stamoulis, N. Al-Dhahir, and A. R. Calderbank, "Further results on interference cancellation and space-time block codes," in Proc. 35th Asilomar Conf. on Signals, Systems, and Computers, Oct. 2001, pp. 257-262. 